\title{
Frameworks para o Planejamento da Gamificação em Contextos Educacionais - Uma revisão da literatura nacional
}

Armando M. Toda - Universidade de São Paulo - armando.toda@gmail.com Wilk Oliveira dos Santos - Universidade de São Paulo - wilk.oliveira@usp.br Ana C. T. Klock - Universidade Federal do Rio Grande do Sul - actklock@inf.ufrgs.br Isabela Gasparini - Universidade do Estado de Santa Catarina isabela.gasparini@udesc.br Ig Ibert Bittencourt - Universidade Federal do Alagoas - ig.ibert@gmail.com Seiji Isotani - Universidade de São Paulo - sisotani@icmc.usp.br

Resumo. A gamificação tem sido bastante utilizada para aumentar o engajamento e a motivação dos estudantes em contextos educacionais, ampliando o interesse por parte de professores e instrutores. No entanto, há um consenso na literatura quanto a necessidade de abordagens sistemáticas (métodos, processos elou frameworks) para apoiar o planejamento da gamificação, de modo que os seus efeitos benéficos sejam alcançados. A literatura identifica alguns estudos internacionais focados em analisar estas abordagens sistemáticas, no entanto, há uma carência de estudos que relatem como o Brasil tem contribuído para o processo de planejamento da gamificação. Diante disso, esse artigo tem por objetivo apresentar um mapeamento sistemático da literatura, visando identificar as abordagens sistemáticas existentes para o planejamento da gamificação em escala nacional. A partir da condução desse mapeamento, foram encontrados 18 estudos onde foram identificados: (a) uma carência no apoio automatizado para o docente; (b) uma classificação dos tipos de personalização utilizados; e (c) a quantidade de elementos de gamificação utilizados. Por meio dos resultados obtidos, foi possivel identificar ainda diversos desafios e oportunidades relacionados a esta área de pesquisa.

Abstract. Gamification has established itself as a technology capable of increasing engagement and motivation in educational contexts, increasing the interest of teachers and instructors. Therefore, there is a consensus in the literature about the need for systematic approaches (methods, processes and / or frameworks) for gamification planning, in order to achieve its beneficial effects. The literature present some international studies focused on analyzing these systematic approaches, however, there is a lack of studies that report how Brazil can contribute to this planning process. Based on the exposed, the objective of this article is to present a systematic mapping of the literature, aiming to identify the existing nationwide approaches to the planning of gamification. From the conduction of this mapping, 18 studies were found. From these studies, we identified: (a) a lack of automated support for the teacher; (b) a classification of the types of personalization used; and (c) the amount of elements used. Through the results, it was also possible to identify several challenges and opportunities related to this area of research.

\section{Introdução}

Nos últimos anos, a gamificação vem se consolidando como uma tecnologia de apoio educacional para auxiliar, engajar e motivar os alunos em ambientes educacionais [Dichev and Dicheva 2017]. Isto tem atraído a atenção de professores, instrutores que, por sua vez, em geral, não possuem tempo, recursos ou conhecimento necessário sobre gamificação para planejar e executar as tarefas gamificadas em seus contextos educacionais [Paula e Fávero 2016, Martí-Parreño et al. 2016, Sánchez-Mena and Martí-Parreño 2016]. 
Para alcançar os efeitos positivos referentes a motivação e engajamento, a gamificação necessita de um bom planejamento, de forma sistemática, [Zichermann and Cunningham 2011, Toda et al., 2018], assim como considerar a personalização [Santos, Bittencourt \& Vassileva, 2018, Monterrat et al. 2014], elementos de jogos utilizados e formas para automatizar o planejamento para auxiliar o docente [Toda et al. 2018]. Além disso, também é discutido na literatura que um bom design gamificado (isto é, a união dos elementos de jogos com os objetivos a serem alcançados) pode evitar efeitos nocivos que a gamificação pode ocasionar, como a indiferença, perda de desempenho, comportamentos indesejados e efeitos declinantes, como a falta de motivação e engajamento [Toda, Valle \& Isotani, 2017].

No entanto, no contexto educacional, sabe-se que existem poucos estudos que analisam abordagens sistemáticas (nesse estudo, adotamos a definição de abordagem sistemática como processos, métodos, estratégias, frameworks e outras formas sequenciais que auxiliassem na implantação) focadas no planejamento da gamificação, além de que grande parte foi criada para atender domínios específicos, sem considerar o professor como parte do processo [Mora et al. 2017]. Além disso, a gamificação tem sido amplamente explorada por meio de estudos secundários (revisões sistemáticas, mapeamentos sistemáticos e revisões da literatura) [Borges et al. 2014, Klock et al. 2015, Toda et al. 2017].

No entanto, grande parte destes estudos focam na gamificação em escala global, que, por restrições de protocolo (e.g., considerar trabalhos apenas no idioma inglês), nos impede de verificar as abordagens sistemáticas que têm sido desenvolvidas em escala nacional, ou seja artigos em português, de veículos de publicação brasileira e publicados por pesquisadores brasileiros e de instituições brasileiras. Por fim, estes estudos não analisam e/ou debatem como essas abordagens sistemáticas de gamificação lidam com aspectos relacionados ao apoio ao professor (e.g., se existe alguma forma de automatizar o processo e/ou o planejamento) e personalização (e.g., se aspectos de personalização da gamificação com base nos usuários são considerados), nem mesmo têm identificado os elementos utilizados (fundamental para desenvolver as estratégias gamificadas [Zichermann \& Cunninghan, 2011]. Baseando-se no exposto, conduzimos um mapeamento sistemático, com o objetivo de identificar estes aspectos relacionados a: (a) apoio computacional ao professor/ instrutor; (b) personalização da gamificação; e (c) elementos utilizados.

\section{Protocolo}

Para a realização desta pesquisa, foram utilizadas as diretrizes propostas por Kitchenham (2004), para o desenvolvimento de estudos secundários. Estas diretrizes são divididas em três fases, sendo: Planejamento, onde são definidas as Questões de Pesquisa (QP), a string de busca, os Critérios de Inclusão (IC) e Exclusão (EC), e as bases de pesquisa; Condução, onde a string de busca é utilizada nas bases de pesquisa selecionadas, e são aplicados os ICs e ECs; e Relatório dos Resultados, onde é realizado uma exposição dos resultados encontrados. Para nortear o nosso trabalho, desenvolvemos a seguinte questão de pesquisa principal "Que abordagens estão sendo desenvolvidas no cenário nacional?", em seguida definimos as questões de pesquisa que seriam respondidas pelo mapeamento, com o intuito de responder a questão principal. No planejamento, foram definidas as seguintes QP.

- QP1: Existem tecnologias computacionais que automatizam a abordagem para apoiar o processo de planejamento nas abordagens analisadas?

- QP2: Existem formas de personalizar a gamificação nas abordagens analisadas? Se existem, quais?

- QP3: Quantos elementos de de jogos são considerados nestas abordagens?

Em seguida, definimos os termos da string de busca. Para as abordagens, optamos pelos termos "abordagem", "estratégia", "framework", "método", "processo", 
"metodologia", "planejamento", "técnica" em combinação com os termos "gamificação" e "gamification". Com relação às bases de pesquisa, foram escolhidas a CEIE $^{1}$, revista RENOTE $^{2}$ e Scopus, por concentrarem grande parte das pesquisas em informática na educação no país, além de serem as bases mais utilizadas em outras revisões realizadas em âmbito nacional [Morais et al. 2017, Lima et al. 2017]. Nossa string foi ainda adaptada e traduzida para a base da Scopus, utilizando os termos "approach", "strategy", "framework", "method", "process", "methodology", "planning", "technique" e "gamification", e os resultados foram filtrados por país de origem.

Após a definição da string e das bases de pesquisa, foram definidos os IC e EC. Foram considerados estudos completos que: (i) apresentam algum tipo de abordagem sistemática para o planejamento da gamificação em contexto educacional; (ii) fossem conduzidos no Brasil; e (iii) fossem desenvolvidos por universidades brasileiras. Como $\mathrm{EC}$, consideramos estudos que: (i) não apresentam algum tipo de abordagem sistemática para o planejamento da gamificação; (ii) não foram conduzidos no Brasil ou por pesquisadores brasileiros; (iii) façam parte da literatura cinza, ou seja, em bases não indexadas e trabalhos de conclusão (como dissertações e teses). $O$ planejamento do protocolo foi validado por 2 especialistas da área de gamificação e em estudos secundários.

\section{Resultados e Discussão}

O processo das buscas ocorreu entre o período de março de 2018 e abril de 2018, sendo conduzido por dois especialistas em gamificação e em estudos secundários. Ao utilizar a string de busca nas bases selecionadas, 61 estudos foram retornados. Em seguida, os especialistas realizaram a leitura dos títulos, resumos e palavras-chaves de todos os artigos, para identificar os estudos candidatos a serem analisados. Caso houvesse uma discrepância, haveria um debate até alcançar um consenso. Após esta análise, foram eleitos 29 estudos candidatos. Os demais 32 artigos foram excluídos por não descreverem ou focarem em uma abordagem sistemática que poderia ser analisada. Por fim, os especialistas realizaram a leitura completa dos 29 estudos candidatos e, após a aplicação dos IC e EC, 18 estudos foram selecionados. Um resumo com as informações dos artigos pode ser encontrado em https://goo.gl/AT98oQ . A Tabela 1 apresenta os 18 estudos analisados e, a partir deles, esta seção discorre sobre como eles foram capazes de responder às questões de pesquisas definidas anteriormente.

\section{Tabela 1: Lista de estudos analisados}

\begin{tabular}{|l|l|l|}
\hline ID & Título & Referência \\
\hline P1 & $\begin{array}{l}\text { An innovative augmented reality educational framework with } \\
\text { gamification to assist the learning process of children with } \\
\text { intellectual disabilities }\end{array}$ & $\begin{array}{l}\text { [Colpani and } \\
\text { Homem 2015] }\end{array}$ \\
\hline P2 & $\begin{array}{l}\text { Requisitos para aplicações gamificadas e de realidade alternada } \\
\text { para alfabetização e aquisição da linguagem em crianças com } \\
\text { síndrome de down }\end{array}$ & $\begin{array}{l}{[\text { Souza et al. }} \\
2017]\end{array}$ \\
\hline P3 & $\begin{array}{l}\text { Um modelo para promover o engajamento estudantil no } \\
\text { aprendizado de programação utilizando gamification }\end{array}$ & $\begin{array}{l}{[\text { Silva et al. }} \\
\text { 2016] }\end{array}$ \\
\hline P4 & $\begin{array}{l}\text { Um processo de gamificação para o ensino superior: experiências } \\
\text { em um módulo de bioquímica }\end{array}$ & $\begin{array}{l}{[\text { Toda et al. }} \\
2016]\end{array}$ \\
\hline P5 & $\begin{array}{l}\text { Game elements in a software engineering study group: a case } \\
\text { study }\end{array}$ & $\begin{array}{l}{[\text { Matsubara }} \\
\text { and Silva } \\
\text { 2017] }\end{array}$ \\
\hline P6 & Gamificação aplicada ao ensino de gerência de projetos de & {$[$ Ferreira et al. } \\
\hline
\end{tabular}




\begin{tabular}{|l|l|l|}
\hline & software & $2016]$ \\
\hline P7 & $\begin{array}{l}\text { Educação empreendedora em uma plataforma tecnológica } \\
\text { ludificada: um estudo de caso }\end{array}$ & $\begin{array}{l}{[\text { Costa et al. }} \\
2017]\end{array}$ \\
\hline P8 & Gamification: a new multimodal approach to education & $\begin{array}{l}{[\text { Orlandi et al. }} \\
2018]\end{array}$ \\
\hline P9 & $\begin{array}{l}\text { BROAD-PLG: modelo computacional para construção de jogos } \\
\text { educacionais }\end{array}$ & $\begin{array}{l}{[\text { Martins et al. }} \\
2015]\end{array}$ \\
\hline P10 & A gamificação aplicada em ambientes de aprendizagem & {$[$ Fardo 2013] } \\
\hline P11 & $\begin{array}{l}\text { Gamification in education: a methodology to identify student's } \\
\text { profile }\end{array}$ & $\begin{array}{l}{[\text { Freitas et al. }} \\
2017]\end{array}$ \\
\hline P12 & $\begin{array}{l}\text { 5W2H framework: A guide to design, develop and evaluate the } \\
\text { user-centered gamification }\end{array}$ & $\begin{array}{l}{[\text { Klock et al. }} \\
2016]\end{array}$ \\
\hline P13 & $\begin{array}{l}\text { Gamificação e avaliação do engajamento dos estudantes em uma } \\
\text { disciplina técnica de curso de graduação }\end{array}$ & $\begin{array}{l}{[\text { Freitas et al. }} \\
2016]\end{array}$ \\
\hline P14 & $\begin{array}{l}\text { Gamificação na educação: um modelo conceitual de apoio ao } \\
\text { planejamento em uma proposta pedagógica }\end{array}$ & $\begin{array}{l}{[\text { Gonçalves et }} \\
\text { al. 2016] }\end{array}$ \\
\hline P15 & $\begin{array}{l}\text { An ontology framework to apply gamification in CSCL scenarios } \\
\text { as persuasive technology }\end{array}$ & $\begin{array}{l}{[\text { Challco et al. }} \\
2016]\end{array}$ \\
\hline P16 & $\begin{array}{l}\text { A link between worlds: towards a conceptual framework for } \\
\text { bridging player and learner roles in gamified collaborative } \\
\text { learning contexts }\end{array}$ & $\begin{array}{l}{[\text { Borges et al. }} \\
2016]\end{array}$ \\
\hline P17 & $\begin{array}{l}\text { Um modelo conceitual para a gamificação de ambientes virtuais de } \\
\text { aprendizagem }\end{array}$ & $\begin{array}{l}{[\text { Klock et al. }} \\
2015]\end{array}$ \\
\hline P18 & $\begin{array}{l}\text { Gamificando a sala de aula: desafios e possibilidades em uma } \\
\text { dundamental }\end{array}$ & $\begin{array}{l}{[\text { Gomes and }} \\
\text { Tedesco, } \\
2017]\end{array}$ \\
\hline
\end{tabular}

\subsection{QP1: Existem tecnologias computacionais para apoiar o processo de planejamento nas abordagens analisadas?}

A QP1 foi responsável por verificar se as abordagens encontradas apresentavam alguma forma de automatização do processo de planejamento da gamificação através de ferramentas computacionais para apoiar o professor ou instrutor. Dentre as 18 abordagens sistemáticas analisadas, nenhuma apresentou tal conceito. Em comparação com os seis frameworks focados no contexto educacional encontrados por Mora et al. (2017), também pode-se observar que nenhum deles tinha o professor como foco ou parte do processo de planejamento, ou apresentava alguma forma de automatizar esse processo.

Esse resultado pode ser justificado pelo fato da área de gamificação ainda ser considerada recente, com o conceito ganhando destaque em 2011 [Deterding et al. 2011]. No entanto, com o crescente interesse por parte de professores e instrutores [Martí-Parreño et al. 2016, Paula and Fávero 2016, Sánchez-Mena and Martí-Parreño 2016], é necessário voltar esforços para apoiar o processo de planejamento de forma automática. Isto pode ser apoiado pelo uso de ferramentas de autoria [Dermeval et al. 2018] ou através do uso de sistemas de recomendação apoiados por dados (Data-driven gamification design). Com isso, acredita-se que seria possível reduzir o esforço e, consequentemente, tempo necessários pelo professor para a implantação de atividades gamificadas em seu contexto educacional. 


\subsection{QP2: Existem formas de personalizar a gamificação nas abordagens analisadas?}

A QP2 buscou identificar aspectos relacionados a personalização e como estes são apresentados nas abordagens sistemáticas analisadas, por exemplo, os tipos de perfil de usuário utilizados. Dos estudos analisados, apenas 7 (38,9\%) apresentaram algum conceito relacionado a personalização. Dentre estes estudos, identificamos três tipos de perfis (Figura 1), sendo:

- Perfis de jogador: utilizam tipologias baseadas em preferências e motivações dos usuários enquanto jogam (P3, P11, P12, P15, P16, P18);

- Perfil demográfico: consideram aspectos como idade e gênero para $\mathrm{O}$ planejamento da gamificação (P11, P18);

- Papéis: são abordadas as partes relacionadas ao processo, como professor e aluno (P17).

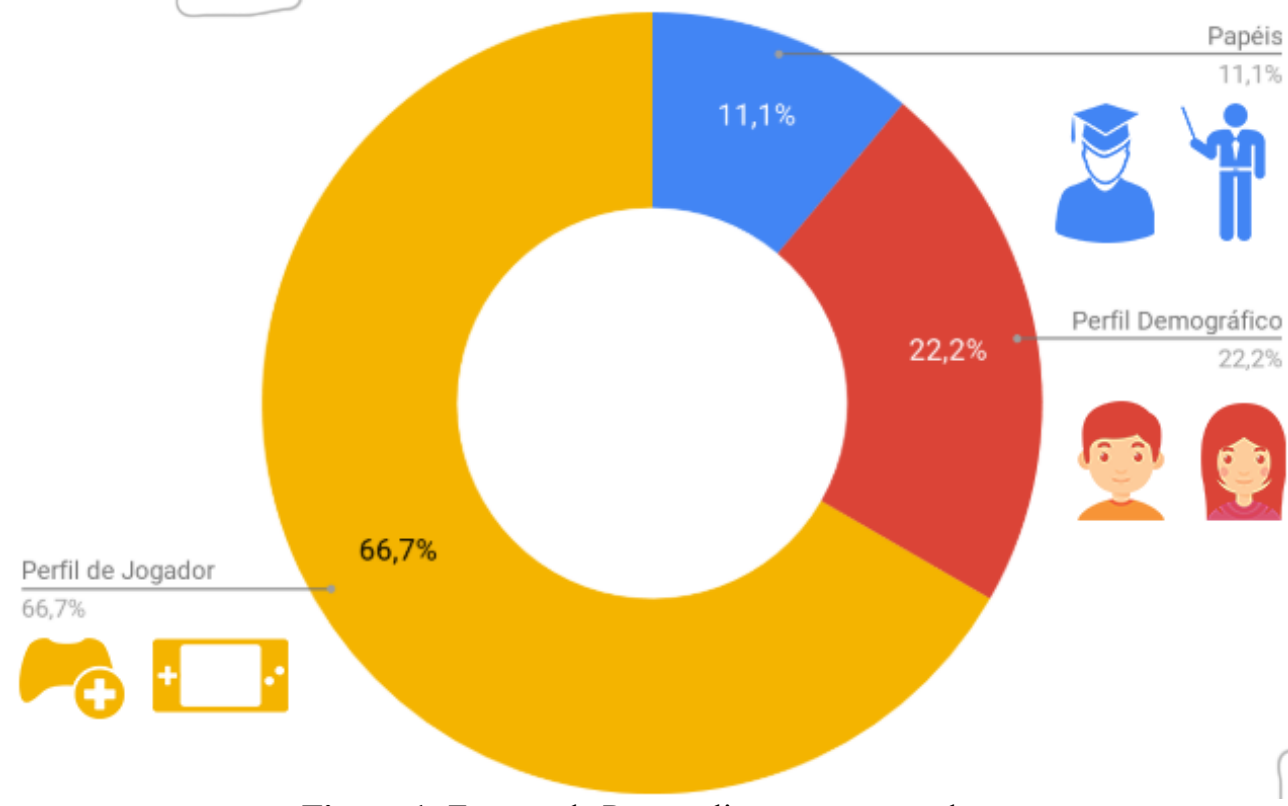

Figura 1: Formas de Personalização encontradas.

A partir da análise destes estudos, pode-se observar que nenhum deles fornece alguma forma de como gamificar o contexto com base no perfil que é citado. Já com relação a identificação dos perfis, esta é sempre realizada através de questionários. Em seguida, realizamos uma comparação com os frameworks (focados no domínio de educação) analisados no estudo de Mora et al. (2017), pode-se observar que todos os frameworks analisados pelos autores também abordam algum tipo de personalização com base no perfil (atributo profiling), porém, apenas um (de seis) desenvolve a proposta de como utilizar elementos de jogos com base no perfil de jogador [Klock et al. 2016].

Esse resultado era esperado, uma vez que a personalização é uma característica essencial a ser considerada em estudos de gamificação [Santos, Bittencourt \& Vassileva, 2018]. Sabe-se que é necessário desenvolver estratégias que se adequem e/ou adaptem ao perfil dos usuários do sistema [Borges et al. 2016]. No entanto, ainda há uma carência de estudos que auxiliem em "como" gerar estas estratégias com base nestes perfis. Também é possível observar que a captação e análise destes perfis ainda é feita de forma manual (e.g., através de questionários), e automatizar este processo poderia também reduzir a carga necessária de tarefas relacionadas ao professor.

3.3 QP3: Quantos elementos de jogos são considerados nas abordagens propostas? 
A QP3 visa identificar como a abordagem identifica e/ou classifica os elementos de jogos utilizados. Dentre os 18 estudos analisados, 7 (38,9\%) não identifica explicitamente quais elementos de jogos são utilizados, $3(16,7 \%)$ criam classificações para os elementos, e os outros 8 estudos $(44,4 \%)$ apresentam uma lista avulsa com os elementos utilizados. Destes 11 estudos que apresentam elementos de jogos em suas descrições, 6 apresentam menos de 10 elementos de jogos (com um mínimo de 6 elementos), enquanto que as 5 demais apresentam mais de 10 elementos de jogos (com um máximo de 21 elementos).

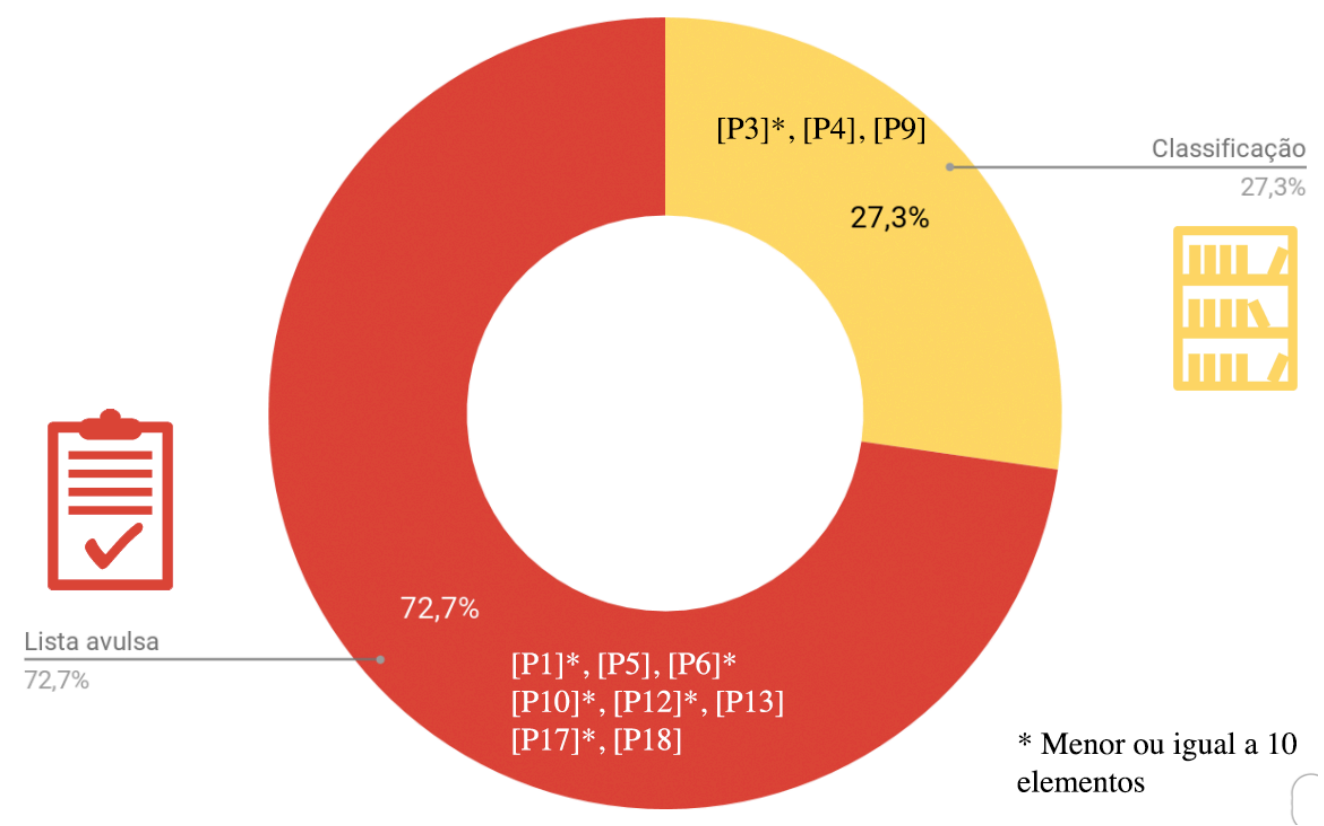

Figura 2: Representação e quantidade de elementos de jogos por abordagem sistemática

Realizamos a análise do número de elementos por abordagem pois esta vem sendo debatida nos últimos estudos relevantes da área [Dichev \& Dicheva, 2017]. Acreditamos que o número de elementos também é uma informação importante para o professor/instrutor escolher uma abordagem, uma vez que o tempo para planejamento pode ser afetado pelo número de elementos que serão analisados e combinados pelo docente. Além disso, vale ressaltar que nas 11 abordagens, que apresentaram elementos de jogos, foram encontrados os elementos Pontos, Níveis e Placares. Esta análise pelo número de elementos não foi realizada por Mora et al. (2017), no entanto, os autores identificam nos estudos se eles abordam o conceito de "ciclos de engajamento". Este conceito define como os elementos de jogos são interligados com as atividades desenvolvidas para engajar o usuário.

Dentre os frameworks analisados por Mora et al. (2017), nenhum abordou os ciclos de engajamento e o mesmo pode ser observado nos 18 estudos analisados nesta revisão. Apesar de alguns conterem uma listagem com os elementos de jogos, não é detalhado como realizar a ligação entre estes elementos para gamificar as atividades a fim de atingir os objetivos propostos. No entanto, alguns dos estudos apresentam instâncias de como podem ser utilizados em seus contextos, apontando possíveis ciclos de engajamento [Toda et al. 2016, Klock et al. 2016].

Quanto ao número de elementos utilizados, acredita-se que a informação é relevante para auxiliar o professor no processo de planejamento, uma vez que alguns dos principais fatores que os afasta são tempo e conhecimento para preparação de recursos [Sánchez-Mena and Martí-Parreño 2016]. Além disso, esse número pode ser insignificante se a proposta sistemática não abordar como utilizá-los através de 
exemplos e/ou instâncias. A discrepância no número de elementos também é encontrada em outros estudos de gamificação, onde ainda há um debate quanto ao melhor número de elementos para alcançar os objetivos propostos [Dichev and Dicheva 2017, Seaborn and Fels 2014].

\section{Desafios, Oportunidades e Direcionamentos de Pesquisa para o Planejamento da Gamificação em Cenários Educacionais}

A partir da análise das abordagens sistemáticas, pode-se verificar que existem ainda desafios a serem superados. Dentre estes, a carência de ferramentas que auxiliem no processo de planejamento da gamificação. Tal desafio impacta diretamente na adoção da gamificação por professores e instrutores, uma vez que há o obstáculo relacionado ao tempo e conhecimento para o planejamento e implantação da gamificação. Diante desse desafio, surge como oportunidade de pesquisa com foco em sistemas de autoria e de recomendação para auxiliar o professor e instrutor a planejarem a gamificação da forma mais adequada. A partir do uso de sistemas de autoria, por exemplo, o professor pode desenvolver suas próprias estratégias gamificadas baseadas em propriedades previamente definidas. Já os sistemas de recomendação podem fornecer estratégias de gamificação com base no contexto em que o professor deseja aplicá-las [Meder et al. 2017].

Outro desafio percebido está relacionado a personalização da gamificação, uma vez que esta é importante para alcançar a motivação individual dos estudantes [Santos, Bittencourt \& Vassileva, 2018, Monterrat et al. 2014]. Apesar de existir uma preocupação quanto ao uso de diferentes perfis e sua extração, ainda não há uma forma sistemática que explique como utilizar os elementos de jogos mais apropriados para perfis específicos, nem mesmo um padrão relacionado aos perfis usados e como gamificar de maneira efetiva para cada um dos perfis. Por fim, não há formas presentes na literatura encontrada de como auxiliar o professor neste processo. Nesse sentido, a área de mineração de dados surge como uma possibilidade computacional para propor maneiras de adequar-se aos diferentes perfis de jogadores e suas condições psicológicas, sociais e humanas. A partir da utilização de técnicas de Mineração de dados, como os algoritmos de predição em bases de dados de sistemas gamificados, pode ser possível identificar estratégias com base nos perfis demográficos dos sistemas, como a preferência de elementos de jogos conforme o gênero dos usuários [Meder et al. 2017].

No que cerne aos elementos de jogos, é possível notar uma discrepância na quantidade de elementos utilizados entre as abordagens analisadas. Tal situação pode prejudicar o entendimento da gamificação por parte dos professores, uma vez que diversos desses elementos possuem conceitos similares (como conquistas e medalhas, ou ranking e placar). Além disso, é válido ressaltar que apesar de apresentarem uma listagem e/ou classificação dos elementos utilizados em cada abordagem, a grande maioria não apresenta diretrizes de como aplicá-los na prática. Dessa forma, surge o desafio de relacionar estes elementos de acordo com sua funcionalidade, objetivo, estratégias de design, entre outros. Nessa direção, o uso de ontologias e outras tecnologias relacionadas a engenharia do conhecimento, podem auxiliar a eliminar redundâncias [Challco et al. 2016]. Além disso, não há estudo empírico que ateste a causalidade do número de elementos com a adoção da gamificação pelo professor ou instrutor.

\section{Limitações, Considerações Finais, e Trabalhos Futuros}

A partir do mapeamento sistemático conduzido, acredita-se que prover evidências de que não há tecnologias computacionais para apoiar o professor no processo de planejamento da gamificação está entre as principais contribuições. Outra contribuição foi a classificação nas formas de personalização abordadas, ressaltando que esta forma de personalização refere-se às características do usuário adotadas para a personalização, e não algoritmos. Também foi realizada uma análise das abordagens sistemáticas quanto 
ao uso de elementos de jogos e de aplicações práticas. Por fim, são propostas oportunidades e direcionamentos com base nos resultados obtidos.

Dentre as ameaças a validade do estudo, destaca-se a abstração de realizar este tipo de estudo secundário, onde podem ocorrer decisões subjetivas. Com o intuito de mitigar possíveis decisões enviesadas, todos os estudos foram discutidos entre os pesquisadores para alcançar um consenso quanto às informações analisadas. Outra ameaça estaria relacionada a ausência de algumas bases de pesquisa comuns na área de computação. Para contornar este viés, utilizamos a Scopus, que indexa diversas bases científicas como Springer e IEEE, filtrando os trabalhos para aqueles desenvolvidos em território nacional.

Como trabalhos futuros, pretende-se analisar as instâncias destas abordagens sistemáticas, com relação às propriedades das avaliações e aplicações na prática (e.g., qual o tipo de avaliação ou como foi realizado o experimento? quais os sujeitos e suas características?). A partir da análise das abordagens sistemáticas presentes, pretende-se desenvolver um framework computacional que auxilie na automatização do planejamento da gamificação, utilizando sistemas de autoria e algoritmos de recomendação, além de um processo que permita a detecção e geração de estratégias de gamificação automáticas com base na personalização do usuário.

\section{Agradecimentos}

Os autores gostariam de agradecer ao CNPq (Processo 307887/2017-0), CAPES, a FAPESP (Processo 2016/02765-2) e a FAPESC (Edital 06/2016, processo 2017TR1755) pelo apoio financeiro.

\section{Referências}

Borges, S. de S., Durelli, V. H. S., Reis, H. M. and Isotani, S. (2014). A systematic mapping on gamification applied to education. In Proceedings of the 29th Annual ACM Symposium on Applied Computing - SAC '14.

Dermeval, D., Albuquerque, J., Bittencourt, I. I., et al. (2018). Amplifying Teachers Intelligence in the Design of Gamified Intelligent Tutoring Systems. In Artificial Intelligence in Education. . Springer International Publishing.

Deterding, S., Dixon, D., Khaled, R. and Nacke, L. (2011). From game design elements to gamefulness: defining gamification. In Proceedings of the 15th international academic MindTrek conference: Envisioning future media environments. ACM.

Dichev, C. and Dicheva, D. (2017). Gamifying education: what is known, what is believed and what remains uncertain: a critical review. International Journal of Educational Technology in Higher Education, v. 14, n. 1, p. 9.

Kitchenham, B. (2004). Procedures for Performing Systematic Reviews. Keele University.

Klock, A. C. T., de Carvalho, M. F., Rosa, B. E. and Gasparini, I. (2015). Análise das técnicas de gamificação em ambientes virtuais de aprendizagem. RENOTE, v. 12, n. 2.

Klock, A. C. T., Klock, da Cunha, L. F. and Gasparini, I. (2015). Um modelo conceitual para a gamificação de Ambientes Virtuais de Aprendizagem. RENOTE, v. 13, n. 1.

Klock, A. C. T., Gasparini, I. and Pimenta, M. S. (2016). 5W2H framework: a guide to design, develop and evaluate the user-centered Gamification. In Proceedings of the 15th Brazilian Symposium on Human Factors in Computing Systems. , IHC '16. ACM.

Lima, D., Netto, J. F. and Menezes, C. D. (2017). Agentes inteligentes e sistemas multiagentes: um mapeamento sistemático focado nas iniciativas brasileiras. In 
Brazilian Symposium on Computers in Education (Simpósio Brasileiro de Informática na Educação - SBIE).

Martí-Parreño, J., Seguí-Mas, D. and Seguí-Mas, E. (2016). Teachers' attitude towards and actual use of gamification. Procedia - Social and Behavioral Sciences, v. 228, p. 682-688.

Meder, M., Plumbaum, T. and Albayrak, S. (2017). A primer on data-driven gamification design. In Proceedings of the Data-Driven Gamification Design Workshop.

Monterrat, B., Lavoué, É. and George, S. (2014). A framework to adapt gamification in learning environments. In European Conference on Technology Enhanced Learning. Springer.

Mora, A., Riera, D., González, C. and Arnedo-Moreno, J. (2017). Gamification: a systematic review of design frameworks. Journal of Computing in Higher Education,

Santos, W. O., Bittencourt, I. I., \& Vassileva, J. (2018). Design of Tailored Gamified Educational Systems Based on Gamer Types. In Anais dos Workshops do Congresso Brasileiro de Informática na Educação (Vol. 7, No. 1, p. 42).

Paula, F. R. D. and Fávero, R. da P. (2016). A gamificação da educação na compreensão dos profissionais da educação. Proceedings of 15th SBGames, p. 14591465.

Sánchez-Mena, A. and Martí-Parreño, J. (2016). Gamification in higher education: teachers' drivers and barriers. Proceedings of the International Conference of The Future of Education, n. July.

Seaborn, K. and Fels, D. I. (2014). Gamification in theory and action: a survey. International Journal of Human-Computer Studies, v. 74, p. 14-31.

Toda, A., Rafael, Y., Cruz, W., Xavier, L. and Isotani, S. (2016). Um processo de gamificação para o ensino superior: experiências em um módulo de bioquímica. In Anais do Workshop de Informática na Escola.

Toda, A. M., Valle, P. H. D., \& Isotani, S. (2017). The Dark Side of Gamification: An Overview of Negative Effects of Gamification in Education. In HEFA 2017: Higher Education for All. From Challenges to Novel Technology-Enhanced Solutions (pp. 143-156). Springer, Cham.

Toda, A. M., da Silva, A. P. and Isotani, S. (2017). Desafios para o Planejamento e Implantação da Gamificação no Contexto Educacional. RENOTE, v. 15, n. 2.

Toda, A. M., Do Carmo, R. M. C., Da Silva, A. P., Bittencourt, I. I. and Isotani, S. (2018). An approach for planning and deploying gamification concepts with social networks within educational contexts. International Journal of Information Management,

Zichermann, G. and Cunningham, C. (2011). Gamification by Design: Implementing Game Mechanics in Web and Mobile Apps. O'Reilly Media; 1 edition.

\section{Apêndice - Referência dos artigos selecionados}

Borges, S. de S., Mizoguchi, R., Durelli, V. H. S., Bittencourt, I. I. and Isotani, S. (2016). A link between worlds: Towards a conceptual framework for bridging player and learner roles in gamified collaborative learning contexts. In Communications in Computer and Information Science. Springer Verlag.

Challco, G. C., Mizoguchi, R. and Isotani, S. (2016). An ontology framework to apply gamification in CSCL scenarios as persuasive technology. Revista Brasileira de Informática na Educação, v. 24, n. 02, p. 67.

Colpani, R. and Homem, M. R. P. (2015). An innovative augmented reality educational framework with gamification to assist the learning process of children with 
intellectual disabilities. In Proceedings of the 6th International Conference on Information, Intelligence, Systems and Applications (IISA). IEEE.

Costa, H., Ferneda, E., and do Prado, H. A. (2017). Educação empreendedora em uma plataforma tecnológica ludificada: um estudo de caso. RENOTE, v. 15, n. 1.

Fardo, M. L. (2013). A gamificação aplicada em ambientes de aprendizagem. RENOTE, v. 11, n. 1 .

Ferreira, L., Inocêncio, A. C., Júnior, P. A. P. and Lopes, M. M. (2016). Gamificação aplicada ao ensino de gerência de projetos de software. In Anais do Workshop de Informática na Escola.

Freitas, S. A. A., Lacerda, A. R. T., Calado, P. M. R. O., Lima, T. S. and Canedo, E. D. (2017). Gamification in education: A methodology to identify student's profile. In Proceedings - Frontiers in Education Conference, FIE. IEEE.

Freitas, S. D., Lima, T., Canedo, E. and Costa, R. L. (2016). Gamificação e avaliação do engajamento dos estudantes em uma disciplina técnica de curso de graduação. In Brazilian Symposium on Computers in Education (Simpósio Brasileiro de Informática na Educação - SBIE).

Gomes, T., \& Tedesco, P. (2017). Gamificando a sala de aula: desafios e possibilidades em uma disciplina experimental de Pensamento Computacional no ensino fundamental. In Anais do Workshop de Informática na Escola.

Gonçalves, L., Giacomazzo, G., Rodrigues, F. and Macaia, B. (2016). Gamificação na Educação: um modelo conceitual de apoio ao planejamento em uma proposta pedagógica. In Brazilian Symposium on Computers in Education (Simpósio Brasileiro de Informática na Educação - SBIE).

Klock, A. C. T., Gasparini, I. and Pimenta, M. S. (2016). 5W2H framework: a guide to design, develop and evaluate the user-centered Gamification. In Proceedings of the 15th Brazilian Symposium on Human Factors in Computing Systems., IHC' '16. ACM.

Martins, G., Campos, F., Braga, R. and David, J. (2015). BROAD-PLG: Modelo Computacional para Construção de Jogos Educacionais. In Brazilian Symposium on Computers in Education (Simpósio Brasileiro de Informática na Educação SBIE).

Matsubara, P. G. F. and Silva, C. L. C. D. (2017). Game Elements in a Software Engineering Study Group: A Case Study. In Proceedings of the 2017 IEEE/ACM 39th International Conference on Software Engineering: Software Engineering Education and Training Track (ICSE-SEET).

Orlandi, T. R. C., Duque, C. G., Mori, A. M. and de Andrade Lima Orlandi, M. T. (2018). Gamification: a new multimodal approach to education. Biblios, n. 70, p. 17-30.

Silva, T., Melo, J. and Tedesco, P. (2016). Um modelo para promover o engajamento estudantil no aprendizado de programação utilizando gamification. In Anais dos Workshops do Congresso Brasileiro de Informática na Educação.

Souza, I. D., Moura, A. and Ghirello-Pires, C. (2017). Requisitos para Aplicações Gamificadas e de Realidade Alternada para Alfabetização e Aquisição da Linguagem em Crianças com Síndrome de Down. In Brazilian Symposium on Computers in Education (Simpósio Brasileiro de Informática na Educação SBIE).

Toda, A., Rafael, Y., Cruz, W., Xavier, L. and Isotani, S. (2016). Um processo de gamificação para o ensino superior: experiências em um módulo de bioquímica. In Anais do Workshop de Informática na Escola. 